\title{
Are We Really That Different From Each Other? The Difficulties of Focusing on Similarities in Cross-Cultural Research
}

\author{
Richard N. Lalonde, Jorida Cila, Evelina Lou, and Robert A. Cribbie \\ York University
}

\begin{abstract}
In this article we argue that there are 2 dominant underlying themes in discussions of strategies for dealing with diversity_-similarity and difference. When we are dealing with social groups, a number of basic psychological processes, as well as popular media and research-based narratives, make it easier to highlight difference rather than similarity. This difference-based approach in research is inherently divisive, but the training that we receive as researchers in the field of psychology has taken us down this path. As a first step, we propose that researchers working in the area of cultural diversity should start making explicit attempts to highlight similarities between groups, even if such similarities are only based on the absence of observed statistical differences. Moreover, if we are going to be serious about demonstrating similarity between groups and certain types of universals in behavior, we should start embracing new approaches to data analyses and consider using statistical procedures that test for equivalence. We illustrate these new techniques using our own data. Finally, we argue that shifting our primary focus from difference to similarity is a worthwhile direction to pursue for successfully managing diversity in multicultural societies.
\end{abstract}

Keywords: cultural diversity, cross-cultural methods, equivalence testing, mate selection, intergenerational conflict

When it comes to managing ethno-cultural diversity in a multicultural setting, different countries have adopted different strategies. The two most widely discussed approaches for managing this diversity have been the models of assimilation and multiculturalism. Moghaddam (2012) has argued that both of these approaches have failed in certain respects. He offers the omnicultural imperative as a third option, proposing that we must first get developing individuals (i.e., children in schools) to focus on human commonalities, and only later in adolescence should notions of group differences and the value of diversity be introduced. Assimilation, multiculturalism, and omniculturalism all deal with the competing themes of similarity and difference. We agree with Moghaddam that highlighting human commonalities has tremendous value, but endorsing this perspective across the board is not an easy road to follow. In this article, we argue that there are psychological (e.g., perception) and societal (e.g., media)
This article was published Online First August 17, 2015.

RichARD N. LALONDE is a professor of psychology at York University in Toronto. He received his $\mathrm{PhD}$ from the University of Western Ontario. His research falls at the intersection of culture, identity, and intergroup relations. Much of his recent research has focused on the bi-cultural identities and experiences of 1st and 2nd generation Canadians.

JoRIDA CILA is a PhD candidate in Social and Personality Psychology at York University. Her research centers around acculturation, identity, and intergroup relations, with a specific focus on ethnic and religious minorities.

Evelina Lou holds a PhD in psychology from York University in Toronto. Her academic research is rooted in the fields of social and cultural psychology. Specific areas of interest include the multi/bicultural experience, racial stereotypes, identity, and well-being. Evelina currently works in a research consulting role at Navigator Ltd., Canada's leading high-stakes public strategy and communications firm.

Robert A. CRIBbie is a professor and member of the Quantitative Methods Program in the Department of Psychology at York University. He received his $\mathrm{PhD}$ in quantitative psychology from the University of Manitoba. His research interests include multiple comparison procedures, robust ANOVA strategies, and equivalence testing.

CORRESPONDENCE CONCERNING THIS ARTICLE should be addressed to Richard N. Lalonde, Department of Psychology, York University, 4700 Keele Street, Toronto ON, Canada, M3J 1P3. E-mail: lalonde@yorku.ca 
processes that may tip the scale in favor of approaches that highlight difference rather than similarity. We also discuss how traditions in social and cultural psychology present challenges to researchers who want to focus on issues of cultural similarity. Finally, we present some of our own research as illustrations of these challenges and offer some strategies for overcoming these obstacles.

\section{The Inevitability of Groups in Basic Psychological Processes}

One of the first challenges of getting individuals to focus on human similarities is that individuals are predisposed to organize their world into groups. A fundamental assumption in the area of perception is that our individual perceptual systems are prewired to focus not only on similarities (e.g., proximity) but also on differences (e.g., categories). These have been written about for quite some time. In his presentation of Gestalt psychology, Köhler (1947) wrote about how we cluster stars in the nighttime sky and observed that "for ages, people have seen the same groups as units, and at the present time children need no instruction in order to perceive the same units" (pp. 141-142). In cognitive psychology, Rosch (1973) wrote about the human propensity to perceive natural categories, and in social psychology, Sherif and Hovland (1961) wrote about how we use assimilation (similarities) and contrast (differentiation) to make social judgments. When individuals in groups first encounter each other, their perceptual and socialcognitive systems thus lead them to notice both similarities and differences. These systems are well in place for individuals living in multicultural societies, where social markers (e.g., language, ethnicity, religion) will inevitably be used to categorize people into different, apparently distinct, groups. The challenge may be to get people, including researchers, to focus on similarities.

\section{The Perpetuation of Groupness (or Difference) in Popular Media Representations}

In his seminal work on group differentiation, Tajfel (1970) provided evidence indicating that individuals will treat each other differently on the basis of their group membership even if the grounds for that group membership are trivial. What he found in the laboratory is commonplace in real intergroup interactions and is magnified even further in situations of extreme intergroup violence such the Holocaust and the Rwandan genocide. Historical examples of this us-them differentiation have often been bolstered by popular media representations of groups. Contemporary representations in multicultural societies may not be as explicitly racist or divisive as older media representations, but they still call on strategies that inherently focus on difference rather than on similarity. We offer two examples from Canadian media.

Our first example concerns the portrayal of Asian Canadians in the media and it has an underlying narrative of difference. In 2010, the English-language national news magazine $\mathrm{Ma}$ cleans published a controversial article titled "Too Asian?" (Findlay \& Kohler, 2010) about the high proportion of Asian students enrolled in Canadian universities. In 2013, a Québécois news magazine, L'actualité, published a piece in which they referred to "Le syndrome de Confucius" (Barlow, 2013). Both stories articulated the view that Asian students are high achievers who focus intensely on academics, but are so achievement-oriented that they have limited social interaction with others and may lack social competence. This narrative stems from the "model minority" stereotype, which views Asians as intelligent, ambitious, and hardworking, but also as potentially unfriendly and sticking only to their own groups (see Lin, Kwan, Cheung, \& Fiske, 2005). Hence, Asians may be perceived as successful, but at a perceived cost to social skills and their ability to integrate. The positive aspects of the stereotype may present a favorable façade, but combined with the negative aspects of this double-edged sword, they are still used to set Asian Canadians apart from other Canadians. These media representations have an underlying message that Asian students are different than the typical Canadian student, who is expected to be much more socially active during the student years.

Our second example concerns a media frenzy surrounding the 2007 murder of 16-year-old Aqsa Parvez at the hands of her Pakistani Canadian father and brother. Much of the media highlighted the role of group membership (e.g., Muslim) rather than focusing on individual 
characteristics of the perpetrators (e.g., need for control) or the dynamics between family members. The overemphasis on group membership will likely detract from the general and serious problem of male and domestic violence and lead to one-dimensional portrayals of Islam as an oppressive culture (Henry, 2010). One particularly controversial article published in Toronto Life, entitled "Girl, Interrupted," (Rogan, 2008), for example, focused on the hijab (Muslim veil) as the catalyst in the murder of Aqsa Parvez. The veil is an accessible visual marker that can be easily used to set some individuals apart (Stemp-Morlock, 2012). From a social psychological perspective, an intense focus on visual markers of group membership (i.e., South Asian and Muslim) in relation to negative connotations (e.g., oppression) form the basis of an availability heuristic (Tversky \& Kahneman, 1973), which people may bring to mind when they think of Muslims or South Asians.

A shared element to both of the above examples is that there is an implied group of social comparison, a group to which the majority of readers might belong (i.e., Canadians of European heritage). Thus they set up a narrative of difference, which is common to many stories that draw lines of social comparison around ethnic or religious groups. There also lies the danger of proscribing a certain type of disposition or behavior because of an individual's particular identity group, which can further solidify these lines of social comparison.

\section{The Narrative of Difference in Social and Cross-Cultural Research in Psychology}

The resounding theme of difference that runs through our perceptual predispositions and popular media constructions also exists in social and cultural psychology. In social psychology, the dominant research paradigm has been the experimental method (for a critique see Rozin, 2001), and in cultural psychology, the dominant paradigm has been a quasi-experimental method (i.e., comparison of naturally occurring groups). Both of these methods draw our attention to differences-either differences between experimental groups (e.g., Asian vs. American cultural worldview prime) or differences between naturally occurring groups (e.g., Asian Americans vs. European Americans) - and exemplify the types of research that are considered normative and valuable in these fields. When trained to conduct psychological research, there is an inherent narrative of the importance of difference. We argue, however, that it is possible to focus on similarities as well as differences. Following is a critical analysis of some of our own research that we use to exemplify the practical challenges associated with demonstrating similarities between social groups.

For the past few years, our research program has been focusing on the experiences of secondgeneration Canadians, and particularly those who are of South Asian and Chinese heritage. These two heterogeneous groups represent the largest visible minority groups in Canada (Statistics Canada, 2008). South Asians are individuals who identify with one of a number of ethnic groups or regions (e.g., Bangladesh, Gujarat, Pakistan, Punjab, Sri Lankan; Lindsay, 2007a). The majority of Chinese in Canada trace their origins to the People's Republic of China, Hong Kong, or Taiwan (Lindsay, 2007b). Both South Asian and Chinese populations grew rapidly in Canada between 1991 and 2001 (Statistics Canada, 2003); these increases can be attributed in part to the growing number of the second-generation within these groups. A general question we have been asking in our research has been whether or not these secondgeneration Canadians differ from their EuroCanadian peers with regard to a variety of social behaviors (e.g., preferred attributes of a life partner, the decision to move out of the family home, interethnic dating). Notice that our general question is focused on difference; it is only recently that we have attempted to take up the challenge of focusing on similarity.

\section{Research Example 1: Culture and Preferred Mate Attributes}

In our first published article on preferred mate characteristics and the influence of culture among South Asian and European Canadian young adults (Lalonde, Hynie, Pannu, \& Tatla, 2004), participants rated 26 attributes that could be important in a potential mate. Our analysis zeroed in on a cluster of 6 "traditional" mate attributes (e.g., family reputation, parents' approval, chastity). Why? Because traditional attributes were theoretically relevant to the arguments made in our article, and because young South Asian Canadians were found to be sig- 
nificantly more likely to prefer these attributes in comparison with European Canadians. What happened to the other 20 attributes? Nothing. They were not even mentioned in a footnote. Looking back at the original data for this paper, there were two striking patterns in the data that we had not written about. First, none of the traditional attributes was evaluated as being very important, relative to other attributes, even for South Asian Canadians. Second, both South Asian and European Canadians highly valued the same types of characteristics in a mate: romantic love, physical attractiveness, a dependable character, and maturity. We did not report the striking similarities between young South Asian and European Canadian respondents, and the editorial review process never asked us to pursue this path. In short, we were following the traditional and rewarded path of reporting and building our research narrative around cultural difference.

Let us move forward 10 years and focus on our most recent article on culture and preferred mate attributes in a multicultural setting (Lalonde, Cila, Lou, \& Giguère, 2013). Here we examined the preferred mate attributes in four groups of young adults from the Toronto area who were of Chinese, South Asian, Italian, or Western European descent. They rated the extent to which they preferred 30 different attributes. Our analysis suggested that 22 of these attributes belonged to three factors. The first factor was labeled congeniality (10 items including mutual attraction/love, physically attractive, dependable character, and emotional stability/maturity). These attributes are the same types of attributes that were the most preferred by participants in our 2004 article, but that we failed to write about. The second factor was called tradition (4 items: similar religion, strong cultural ties, similar culture, and parental approval). The third factor was labeled status (8 items including social status, family reputation, and good financial prospect). A summary of the descriptive results from this study are presented in Figure 1.

If you are trained as a psychologist, where do you direct your attention when you look at Figure 1? We would wager that many a research psychologist would focus on the attributes related to tradition because these are the ones that are associated with the greatest group differ-

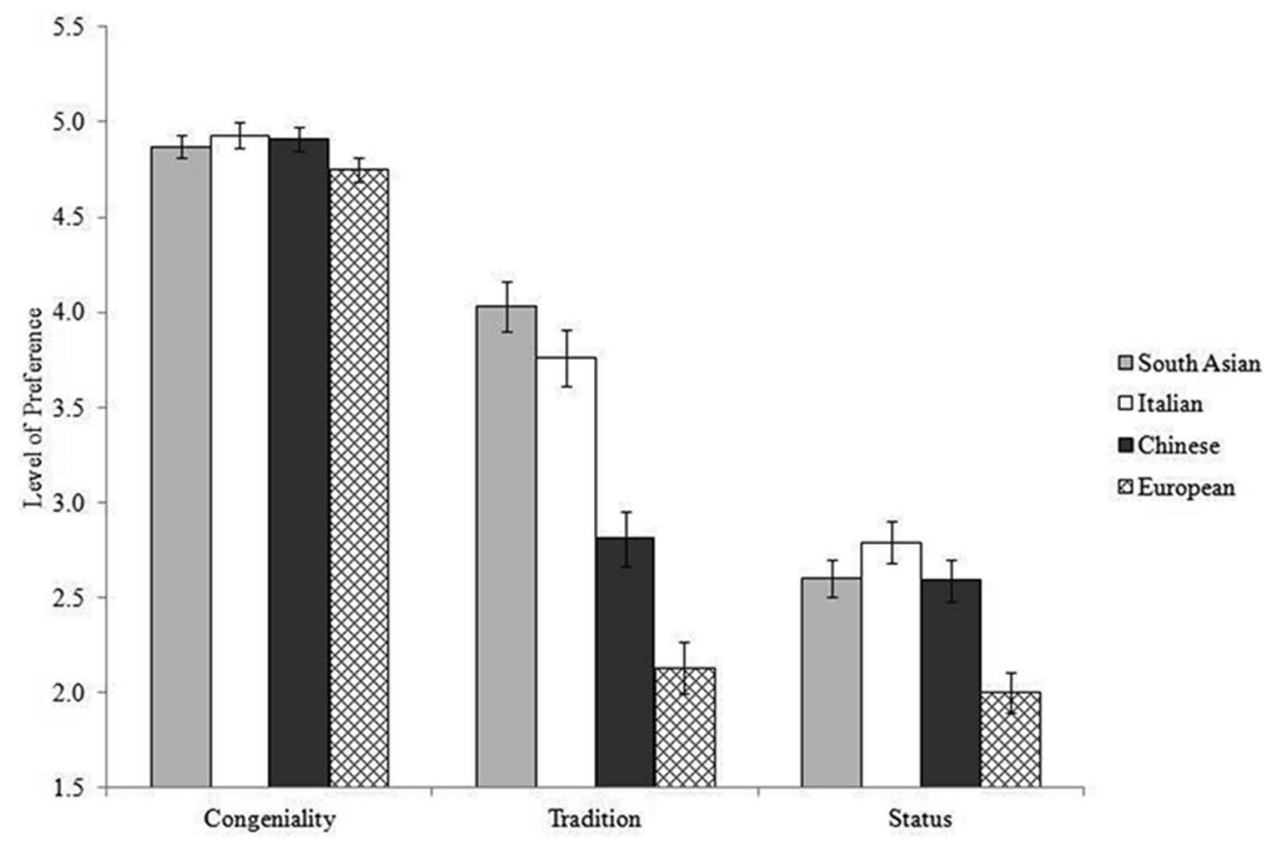

Figure 1. Preferred mate attributes among South Asian, Italian, Chinese, and European Canadian young adults. 
ences. In fact, there was a significant relationship between cultural group membership and a preference for traditional attributes. Young Canadians of South Asian and Italian heritage were significantly more likely to prefer traditional attributes in a mate than were Chinese Canadians, who in turn were more likely to prefer such attributes than Canadians of Western European origin (see Lalonde et al., 2013).

The most striking feature of the data in Figure 1, however, might be the remarkable cultural similarity with regard to preference for congenial attributes in a mate. The scale accompanying the items ranged from 0 (irrelevant or unimportant) to 6 (required or indispensable). Our participants, therefore, were in strong agreement about what they most wanted in a life partner (e.g., dependability) and this appears to be uninfluenced by culture. But how can researchers demonstrate group similarity using more rigorous criteria akin to the procedures that they use to demonstrate difference?

\section{Demonstrating Similarity}

The quest for finding empirical differences in psychological research is evidenced in the dominance of difference-based statistical methods (e.g., $t$ tests, analysis of variance). Researchers are all too familiar with how such procedures function and how to apply them to their datathey often engage in "statistical rituals" and make automatic judgments in their application of the statistical routines (see Gigerenzer, 2004, for a critique). Moreover, the software that is readily available to researchers facilitates the use of difference-based analyses. Although difference-based tests are often practical and welldeveloped for traditional hypothesis testing, they do not allow us to make conclusions about group similarity; failing to reject the null hypothesis does not indicate that group means are similar or equivalent. There are, however, some statistical procedures that can be used to indicate between-groups similarity.

One strategy that has been used to demonstrate group similarities has been to look at effect sizes derived from meta-analyses. Hyde (2005) adopted such a strategy to test the gender similarities hypothesis ("males and females are similar on most, but not all, psychological variables" p. 581). She predicted that most of the gender differences that have been observed in the psychological literature would be associated with effect sizes $(d)$ that would be either closeto-zero $(d \leq 0.10)$ or small $(0.11<d<0.35)$. When examining the data obtained from 46 meta-analyses, she found that $78 \%$ of observed effect sizes were in the close-to-zero or small range. Large gender differences were only found for some motor (e.g., throwing velocity) and sexual (e.g., masturbation) behaviors. Hyde claimed support for the gender similarities hypothesis on the basis of these findings. When focusing exclusively on academic performance in math, Hyde reports a mean effect size of zero, once again supporting the gender similarities hypothesis (Hyde, Lindberg, Linn, Ellis, \& Williams, 2008).

More recently, quantitative psychologists are beginning to direct their attention to using equivalence-based procedures (e.g., Cribbie, Gruman, \& Arpin-Cribbie, 2004). These procedures offer more valid tests of similarity between groups and have been used in other disciplines (e.g., pharmaceutical studies) for some time (e.g., Schuirmann, 1987). In psychological research, Ball, Cribbie, and Steele (2013) used an equivalence testing procedure to test Hyde's gender similarity hypothesis with regard to mathematical ability. Using a large body of SAT-M scores collected over a 14-year period, they were able to demonstrate that performance of women and men on the math SAT were statistically equivalent. We will apply a procedure similar to the one used by Ball et al. (2013) to our preferred mate attributes data and test the hypothesis of cultural equivalence for a preference for congenial attributes in a mate.

\section{Testing for Group Similarity in Mate Preferences for Congeniality}

The descriptive statistics for the congeniality measure presented in Figure 1 reveal a striking pattern of similarity across the four cultural groups (South Asian, $n=120, M=4.86, S D=$ .69 ; Italian, $n=92, M=4.93, S D=.64$; Chinese, $n=95, M=4.91, S D=.65$; European, $n=107, M=4.75, S D=.58$ ). Not surprisingly, when comparing these means using an ANOVA procedure, no difference is found, $F(3,410)=1.56, p=.20$. To demonstrate that these groups are statistically equivalent on this measure, we used a robust version 
of the method outlined by Wellek (2010) and Koh and Cribbie (2013) for evaluating the equivalence of two or more independent groups, even when population variances are unequal. Cribbie, Gruman, and Arpin-Cribbie (2004) and Koh and Cribbie (2013) have demonstrated that this approach is superior to conducting multiple two-sample equivalence tests when more than two groups are being compared.

A good way to frame the problem of equivalence testing would be to ask "what is the largest difference between the population means that would not be meaningful in the context of the study?" (Ball et al., 2013, p. 150). In other words, to what extent can groups differ on a construct and still be considered "not different" from each other? The null hypothesis in this case is that the difference in the means (quantified using the statistic $\psi^{2}$ ) exceeds some critical difference (quantified by $\varepsilon^{2}$; i.e., $\psi^{2} \geq$ $\varepsilon^{2}$ ), whereas the alternate hypothesis is that the difference in the means is smaller than the critical difference (i.e., $\psi^{2}<\varepsilon^{2}$ ). In essence, when using equivalence testing, a researcher sets critical bounds a priori and there is an explicit test of whether a difference between groups falls within these bounds. There is a challenge in determining the appropriate equivalence interval, however, as there is no obvious magic quantity that represents a true difference.

To set the equivalence interval, we followed Wellek's (2010) recommendations. Note that $\varepsilon=.25$ can be equated (in the two sample case) to a Cohen's $d$ of approximately .37, and $\varepsilon=.5$ can be equated (again in the two sample case) to a Cohen's $d$ of approximately .74. If we convert these to Cohen's $f$ for the current four group situation, that is an $f=.13$ for $\varepsilon=.25$ and $f=$ .26 for $\varepsilon=.5$. In both cases we are talking about less than $6 \%$ of the variability being due to cultural group membership. We adopted an $\varepsilon=.5$ for the current data set.

To conduct our analysis, we could not rely on a standard software package like SPSS or SAS. The data were analyzed using $R$, an open-source statistical program that can be freely obtained. The $R$ function that was used for this analysis can be found at http://www.psych.yorku.ca/ cribbie/rfunc.html. The results indicate that our test statistic, $\psi^{2}=.052$, is less than the critical $\psi^{2}\left(.129\right.$, which is based on $d f_{\text {num }}=3, d f_{\text {den }}=$ $223.59, n c p=26.25$, where $n c p$ is the noncentrality parameter calculated as the mean group size multiplied by $\varepsilon^{2}$ ). Thus, we can reject the null hypothesis that the means are not equivalent, and side with the alternative hypothesis of group mean equivalence.

On the basis of the above analysis, we now have statistical evidence of the equivalence of the four groups in terms of their preferences for congenial attributes in a mate. In sum, young Canadians of South Asian, Italian, Chinese, or European heritage are all in strong agreement that they want a life partner who has attributes such as love, dependability, and maturity. We also have an example of one tool that psychologists can use to demonstrate equivalence or group similarity. Such procedures could be applied to other data that have been collected in the context of cross-cultural comparisons and can be particularly valuable for studies comparing groups living in the same multicultural nation where problems of measurement equivalence are less likely a concern.

\section{Research Example 2: Culture, Gender, and Intergenerational Conflict}

We recently collected some data to determine whether intergenerational cultural conflicts are more gendered for particular cultural groups. Samples of emerging adults $\left(M_{\mathrm{age}}=19.64\right)$ from South Asian $(n=250)$ and European $(n=$ 279) Canadian groups were given Chung's (2001) 24-item Intergenerational Conflict Inventory, which was developed using a broad sample of Asian Americans. This survey assessed the reported extent of parent-child conflict in three domains: family expectations (e.g., "following cultural traditions"; 11 items; $\alpha=$ .81), career and education (e.g., "emphasis on success and materialism"; 10 items; $\alpha=.84$ ), and dating and marriage (e.g., "when to begin dating"; 3 items; $\alpha=.85$ ). Items were rated using a 7-point scale where 1 was labeled no conflict, 4 was labeled some conflict, and 7 was labeled $a$ lot of conflict.

To provide some context for this research example, we briefly highlight some of the literature. Previous research has shown that parentchild conflict tends to be higher among immigrant compared with nonimmigrant families. In particular, Farver, Xu, Bhadha, Narang, and Lieber (2007) found that Indian American adolescents reported higher generalized conflict 
with their parents compared to their European American peers. Thus, we expected to see higher levels of conflict among South Asian Canadians than European Canadians across all three conflict domains. We could also expect, on the basis of some prior qualitative research (Gupta, Johnstone, \& Gleeson, 2007; Talbani \& Hasanali, 2000) as well as media reports (e.g., the Aqsa Parvez story mentioned earlier), that this cultural effect might be moderated by gender: young South Asian women may report more conflict than their male counterparts, particularly in the area of dating and marriage.

The basic descriptive data for this study can be found in Figure 2. Again, which part of this Figure would your attention likely be drawn to if you were trained as a psychologist? Such training would likely lead you to focus on the difference between South Asian Canadians and European Canadians in reported conflict in the area of dating and marriage. This is in fact the biggest difference and the traditional approach would be to test to see whether this and other mean differences are statistically significant.

The data were therefore analyzed using the traditional analysis of variance approach with culture and gender serving as the independent variables and the three conflict scales as the dependent measures. As predicted, there was a main effect of culture for each of the conflict measures: family, $F(1,465)=5.37, p=.02$, career, $F(1,465)=6.40, p=.01$, and dating and marriage, $F(1,463)=7.56, p=.006$. However, there were no statistically significant gender main effects (family, $F(1,465)=0.78$, $p=.38$; career, $F(1,465)=0.003, p=.96$; and dating and marriage, $F(1,463)=0.012, p=$ $.011)$ or culture by gender interaction effects (family, $F(1,465)=0.20, p=.89$; career, $F(1$, $465)=0.12, p=.73$, and dating and marriage, $F(1,463)=0.33, p=.57)$ for any of the domains of conflict. The overall pattern of the data (focusing on the effect of both independent variables) may thus reflect more a pattern of similarity (or at least nondifference) than of difference. If these were the only data that we collected in our study, the normative approach would be to write a paper that plays up significant cultural group differences in the different domains of intergenerational conflict. But how big or meaningful are these differences? Let us address this question by focusing on the Co-

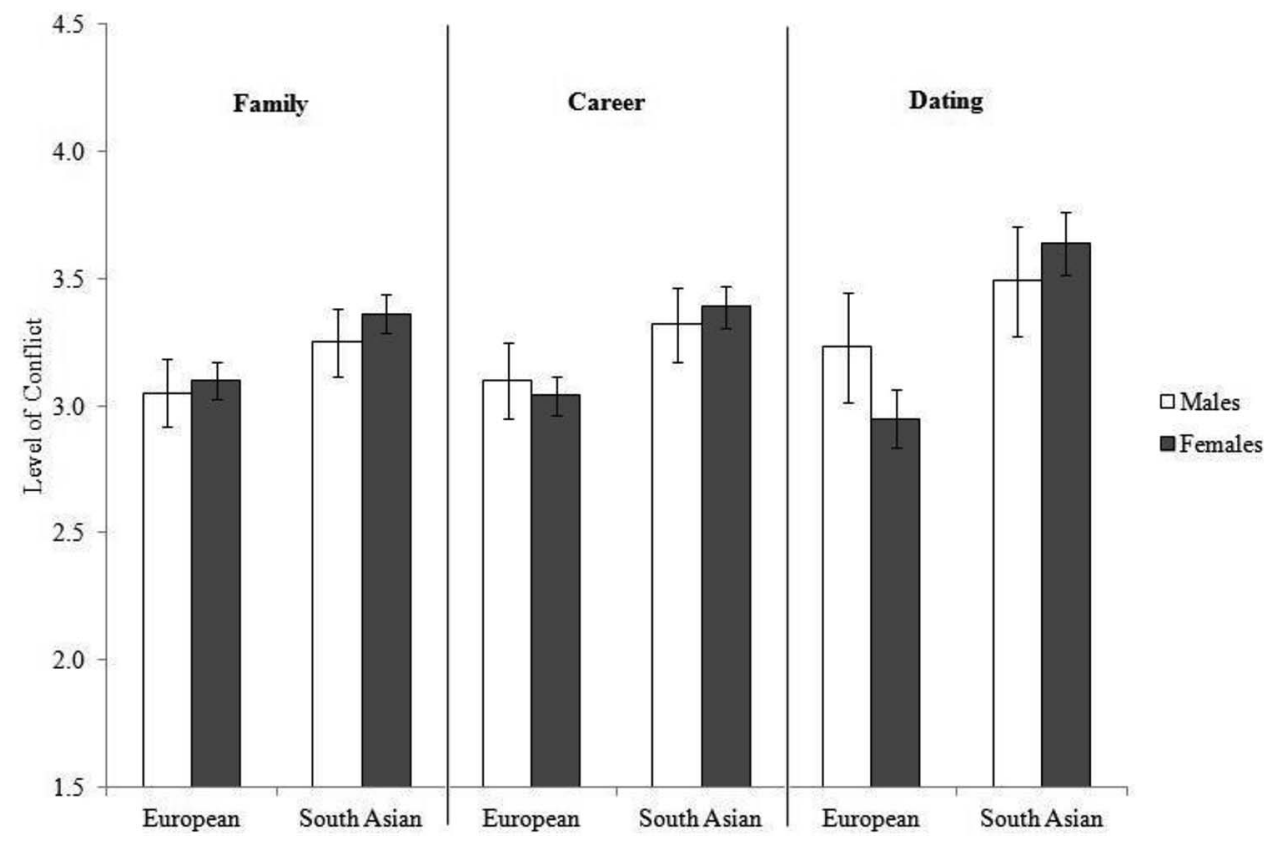

Figure 2. Intergenerational conflict in three domains among European and South Asian Canadian men and women. 
hen's $d$ coefficients associated with the observed cultural differences.

\section{Culture Effects-Difference and Similarity}

The means and tests of difference between South Asian Canadians and European Canadians on the conflict measures in the domains of family, career, and dating and marriage were respectively as follows: family, $M=3.33$ versus $M=3.08, t(467)=2.62, p=.009, d=.24$; career, $M=3.39$ versus $M=3.04, t(467)=$ $3.22, p=.001, d=.30$; and dating and marriage, $M=3.59$ versus $M=3.02, t(465)=$ $3.64, p=.000, d=.34$. If we adopt the same criteria as did Hyde (2005) when testing the gender similarities hypothesis, the above cultural differences could be described as small $(0.11<d<0.35)$. Hyde would argue that such effects may be suggestive of similarity because they are quite small.

Although it can be argued that criteria for defining small or large effects are rather arbitrary, the point we would like to make is that an honest presentation of group differences involves at the very least a minimal discussion of the magnitude and meaningfulness of these differences. If we return to the visual presentation of the data in Figure 2, we can contextualize our observed "statistical differences." All of the means reported in the above analyses of cultural differences fall between 3.0 and 3.6 on a 6-point scale. Given that the midpoint on the scale is 3.5 , we can state that there are only moderate levels of conflict for all groups. None of the group means for the conflict measures are particularly high and none are particularly low, thus none of the cultural group differences could be described as "striking" or "big." We can only comfortably state that there are small cultural differences between South Asian Canadians and European Canadians in their reported levels of intergenerational conflict and that the overall pattern suggests that they are similarly reporting moderate levels of conflict.

\section{Gender Effects-Difference and Similarity}

There is a greater challenge in discussing the pattern of means associated with gender in the above analyses. Because no main effects or interactions involving gender were found in the results from the analysis of variance, we have no statistical support for the hypothesis that gender interacts with culture in predicting intergenerational conflict. Some researchers would point to the pattern of means and report that although there were no significant effects, the means were in the predicted direction (particularly for reported intergenerational conflict around the issue of dating and marriage).

So what can be said about the similarity of these young women and men regarding their reported intergenerational conflicts? We cannot equate the absence of a statistical difference with similarity (i.e., support the null hypothesis). We cannot test for equivalence as we did in our first research example, as there are no theoretical or empirical grounds to do so, based on the existing literature. Nonetheless, the somewhat similar results for males and females in the South Asian sample is particularly interesting because it undermines popular stereotypes about gendered conflicts in South Asian families. It can be argued, therefore, that a researcher should be obliged to at least point out the similarity in the pattern of responses from men and women, the same way that research psychologists often point out patterns of difference in the absence of statistically significant results.

\section{Conclusion}

While strategies like the omnicultural approach (Moghaddam, 2012) offer intriguing options for managing diversity in a multicultural context, one must be realistic in acknowledging the dispositional and practical challenges of focusing primarily on similarities. Taking the first steps to identifying similarities across groups requires stepping back from traditional approaches in the field of psychology and overcoming our inherent tendencies to perceive difference.

There have been prior critical analyses of this "culture of difference" in psychological research. Lamiell (2003), for example, has questioned whether a focus on individual and group differences has exacerbated a culture of separation, and consequently whether it is undermining rather than enhancing communities. $\mathrm{He}$ argues that this is the case, and that for tolerance to work, people need to see others as individuals and to appraise their own as well as other peoples' values without relying on stereotypes. 
Lamiell further suggests that "a personalistic conception of individuality" - one that is not concerned with comparisons and differences-is ideal. He gives the example of adopting higher-order identities, such as a human being, as opposed to ethnicity-based categorizations. In the veins of peace research and peace journalism (see Galtung \& Fischer, 2013; Kempf, 2003), which seek to understand not only why conflicts arise in societies but also why peace can also exist in so many societies, it may be worthwhile to place our focus more on commonalities that are positive rather than on differences that lead to separation.

Given that we are working within a tradition of psychology where the normative approach reinforces a research culture of difference, the challenge of integrating similarity into our work is daunting, and we hope that we have provided some examples of how this can be done. In addition to promoting the development and appropriate use of statistical equivalence testing, we also need to encourage journal editors to be open to a lens of similarity in the presentation of data. Furthermore, being able to purposefully consider and promote similarity also means that one must understand and take into account the local context-no two countries share the same sociohistorical background, and even within a single country we cannot assume identical relationships between the majority and each cultural group (e.g., minority, immigrant) living in that country. Thus, there may not be a single recipe for successfully managing diversity, but bringing attention to the things that connect us together, rather than those that set us apart, is a promising start.

\section{References}

Ball, L. C., Cribbie, R. A., \& Steele, J. R. (2013). Beyond gender differences: Using tests of equivalence to evaluate gender similarities. Psychology of Women Quarterly, 37, 147-154. http://dx.doi .org/10.1177/0361684313480483

Barlow, J. (2013, June 5). Voici les nouveaux SinoQuébécois [Here are the new Asian-Québécois]. L'actualité. Retrieved November 14, 2013, from http://www.lactualite.com/societe/voici-lesnouveaux-sino-quebecois/

Chung, R. H. G. (2001). Gender, ethnicity, and acculturation in intergenerational conflict of Asian American college students. Cultural Diversity and Ethnic Minority Psychology, 7, 376-386.
Cribbie, R. A., Gruman, J. A., \& Arpin-Cribbie, C. A. (2004). Recommendations for applying tests of equivalence. Journal of Clinical Psychology, 60, 1-10. http://dx.doi.org/10.1002/jclp.10217

Farver, J. M., Xu, Y., Bhadha, B. R., Narang, S., \& Lieber, E. (2007). Ethnic Identity, acculturation, parenting beliefs, and adolescent adjustment: A comparison of Asian Indian and European American families. Merrill-Palmer Quarterly, 53, 184 215. http://dx.doi.org/10.1353/mpq.2007.0010

Findlay, S., \& Kohler, N. (2010, November 10). The enrollment controversy: Worries that efforts in the U.S. to limit enrolment of Asian students in top universities may migrate to Canada. Macleans. Retrieved November 13, 2010, from http://www2 .macleans.ca/2010/11/10/too-asian/

Galtung, J., \& Fischer, D. (2013). Johan Galtung: Pioneer of peace research. In H. G. Brauch (Ed.), Springer briefs on pioneers in science and practice (Vol. 5). New York, NY: Springer.

Gigerenzer, G. (2004). Mindless statistics. Journal of Socio-Economics, 33, 587-606. http://dx.doi.org/ 10.1016/j.socec.2004.09.033

Gupta, V., Johnstone, L., \& Gleeson, K. (2007). Exploring the meaning of separation in secondgeneration young South Asian women in Britain. Psychology and Psychotherapy: Theory, Research and Practice, 80, 481-495. http://dx.doi.org/ 10.1348/147608307X173986

Henry, L. (2010). Minorities in Canadian media: Islam and the case of Aqsa Parvez. The Laurier M. A. Journal of Religion and Culture, 2, 39-56.

Hyde, J. S. (2005). The gender similarities hypothesis. American Psychologist, 60, 581-592. http://dx .doi.org/10.1037/0003-066X.60.6.581

Hyde, J. S., Lindberg, S. M., Linn, M. C., Ellis, A. B., \& Williams, C. C. (2008). Diversity. Gender similarities characterize math performance. Science, 321, 494-495. http://dx.doi.org/10.1126/science .1160364

Kempf, W. (2003). Constructive conflict coverage-A social-psychological research and development program. Conflict \& Communication Online, 2. Retrieved from www.cco.regener-online.de

Koh, A., \& Cribbie, R. (2013). Robust tests of equivalence for $k$ independent groups. British Journal of Mathematical and Statistical Psychology, 66, 426-434.

Köhler, W. (1947). Gestalt psychology: An introduction to new concepts in modern psychology. New York, NY: Liveright.

Lalonde, R. N., Cila, J., Lou, E., \& Giguère, B. (2013). Delineating groups for cultural comparison in a multicultural setting: Not all Westerners should be put into the same melting pot. Canadian Journal of Behavioural Science/Revue canadienne des sciences du comportement, 45, 296-304. http://dx.doi.org/10.1037/a0034257 
Lalonde, R. N., Hynie, M., Pannu, M., \& Tatla, S. (2004). The role of culture in interpersonal relationships: Do second generation South Asian Canadians want a traditional partner? Journal of Cross-Cultural Psychology, 35, 503-524. http://dx .doi.org/10.1177/0022022104268386

Lamiell, J. T. (2003). Beyond individual and group differences: Human individuality, scientific psychology, and William Stern's critical personalism. Thousand Oaks, CA: Sage.

Lin, M. H., Kwan, V. S. Y., Cheung, A., \& Fiske, S. T. (2005). Stereotype content model explains prejudice for an envied outgroup: Scale of antiAsian American Stereotypes. Personality and Social Psychology Bulletin, 31, 34-47. http://dx.doi .org/10.1177/0146167204271320

Lindsay, C. (2007a). Profiles of ethnic communities in Canada: The South Asian community in Canada, 2001. Ottawa, ON: Statistics Canada.

Lindsay, C. (2007b). Profiles of ethnic communities in Canada: The Chinese community in Canada, 2001. Ottawa, ON: Statistics Canada.

Moghaddam, F. M. (2012). The omnicultural imperative. Culture \& Psychology, 18, 304-330. http:// dx.doi.org/10.1177/1354067X12446230

Rogan, M. (2008, December). Girl, interrupted. Toronto Life, 42, 52-59.

Rosch, E. H. (1973). Natural categories. Cognitive Psychology, 4, 328-350. http://dx.doi.org/ 10.1016/0010-0285(73)90017-0

Rozin, P. (2001). Social psychology and science: Some lessons from Solomon Asch. Personality and Social Psychology Review, 5, 2-14. http://dx .doi.org/10.1207/S15327957PSPR0501_1

Schuirmann, D. J. (1987). A comparison of the two one-sided tests procedure and the power approach for assessing the equivalence of average bioavailability. Journal of Pharmacokinetics and Biophar- maceutics, 15, 657-680. http://dx.doi.org/10.1007/ BF01068419

Sherif, M., \& Hovland, C. I. (1961). Social judgment: Assimilation and contrast effects in communication and attitude change. New Haven, CT: Yale University Press.

Statistics Canada. (2003). Ethnic diversity survey: Portrait of a multicultural society. Statistics Canada Catalogue no. 89-593-XIE. Ottawa. September 2003. Minister of Industry. Retrieved from http://www.statcan.gc.ca/pub/89-593-x/89-593x2003001-eng.pdf

Statistics Canada. (2008). Canada's ethnocultural mosaic, 2006 census: National picture. Statistics Canada Catalogue no. 97-562-XWE2006001. Ottawa. April 9. Analysis Series, 2006 Census. Retrieved August 11, 2008, from http://www12 .statcan.ca/english/census06/analysis/ethnicorigin/ south.cfm

Stemp-Morlock, L. (2012). Blinded by the veil. Textiles and Politics: Textile Society of America 13th Biennial Symposium Proceedings, Washington, DC.

Tajfel, H. (1970). Experiments in intergroup discrimination. Scientific American, 223, 96-102. http:// dx.doi.org/10.1038/scientificamerican1170-96

Talbani, A., \& Hasanali, P. (2000). Adolescent females between tradition and modernity: Gender role socialization in South Asian immigrant culture. Journal of Adolescence, 23, 615-627. http:// dx.doi.org/10.1006/jado.2000.0348

Tversky, A., \& Kahneman, D. (1973). Availability: A heuristic for judging frequency and probability. Cognitive Psychology, 5, 207-232. http://dx.doi .org/10.1016/0010-0285(73)90033-9

Wellek, S. (2010). Testing statistical hypotheses of equivalence and noninferiority (2nd ed.). New York, NY: CRC Press. http://dx.doi.org/10.1201/ EBK1439808184 